\title{
Mucocutaneous Leishmania tropica infection in a dog from a human cutaneous leishmaniasis focus
}

\author{
Gad Baneth ${ }^{1 *}$, Doni Zivotofsky², Yaarit Nachum-Biala', Daniel Yasur-Landau' and Ana-Maria Botero ${ }^{3}$
}

\begin{abstract}
Background: Leishmania tropica is a causative agent of cutaneous leishmanaisis in the Middle East, North Africa and parts of southeastern Europe. Although transmission of L. tropica has been reported as anthroponotic, in Israel it was found to have a zoonotic pattern.

Findings: A one year old male Pekingese dog from Maale Adumim, a focus of L. tropica human cutaneous leishmaniasis near Jerusalem, was presented by its owner with a large proliferative red mucocutaneous lesion on the lip between the mouth and nose. Physical examination and a biochemistry panel were normal and a complete blood count showed mild leukocytosis with lymphocytosis and eosinophilia. A biopsy of the lesion was suggestive of the presence of Leishmania organisms. Serology for Leishmania sp. by ELISA was positive and an aspirate from the lesion showed a large number of Leishmania amastigotes. ITS1-HRM-PCR of the lesion was positive and sequencing indicated that infection was caused by L. tropica, which was also cultured from the lesion. Blood PCR was negative. The dog responded well to allopurinol treatment and its lesion shrunk considerably within one month of therapy and healed after two months.

Conclusions: Only a few cases of dog infection with L. tropica have been described to date. They were reported from Morocco and Iran and involved infection of visceral organs. This is the first report of focal mucocutaneous L. tropica infection in a dog and its response to anti-leishmanial treatment. Domestic and wild canines should be evaluated for being possible animal reservoirs for human L. tropica infection in endemic areas or merely accidental hosts.
\end{abstract}

Keywords: Leishmania tropica, Mucocutaneous leishmaniasis, Dog, Israel, Allopurinol

\section{Findings}

A one year old male Pekingese dog from Maale Adumim, a focus of Leishmania tropica human cutaneous leishmaniasis near Jerusalem, Israel, was presented by its owner to a local veterinary clinic with a large proliferative red mucocutaneous lesion on the upper lip extending all the way between the nose and mouth (Figure 1). Physical examination was otherwise normal and the dog was initially treated with the antibiotic cephalexin at $20 \mathrm{mg} / \mathrm{kg}$ orally every 12 hours for 10 days and with the antifungal itraconazole at $5 \mathrm{mg} / \mathrm{kg} /$ day orally for 10 days for suspected bacterial dermatitis or fungal infection. After no improvement was observed, a full thickness biopsy of the lesion was taken under short general anesthesia and

\footnotetext{
*Correspondence: gad.baneth@mail.huji.ac.il

${ }^{1}$ School of Veterinary Medicine, Hebrew University, P.O. Box 12, Rehovot 76100, Israel

Full list of author information is available at the end of the article
}

submitted for histological evaluation. Microscopically, the skin had extensive epidermal ulceration with underlying heavy dermal infiltration of numerous reactive macrophages interspersed with neutrophils and aggregations of plasma cells. Macrophages contained numerous basophilic oval organisms, measuring approximately 1 to $2 \mu \mathrm{m}$, interpreted as Leishmania amastigotes (Figures 2 and 3). Amastigotes were also present in fibroblasts as previously reported for Leishmania infantum [1] (Figure 3). Following the presumptive diagnosis of leishmaniasis, a complete blood count (CBC) and serum biochemistry panel were taken, as well as serology for L. infantum by ELISA as previously described [2] and blood for PCR using the ITS1-PCR- high resolution melt (HRM) analysis [3]. The lesion was aspirated for cytology, ITS1-PCR-HRM, and parasite culture in NNN slants overlaid with Schneider's Drosophila medium as previously described [2]. 


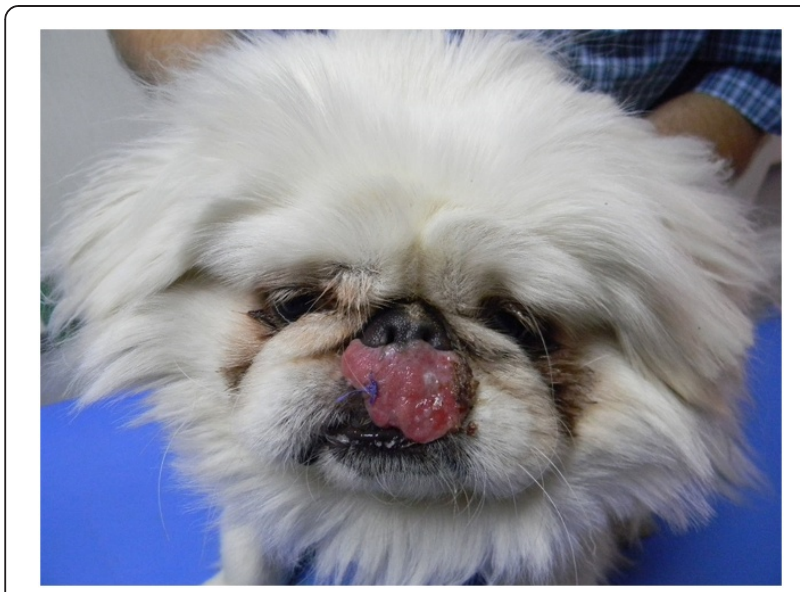

Figure 1 Mucocutaneous Leishmania tropica in the dog. Dog showing mucocutaneous lesion caused by Leishmania tropica before treatment.

A blood count showed a leukocytosis $\left(17.9 \times 10^{3}\right.$ leukocytes/ $\mu$ l; reference 5.2-13.9 $\times 10^{3}$ ) comprised of lymphocytosis $\left(7.87 \times 10^{3}\right.$ lymphocytes $/ \mu \mathrm{l}$; reference $\left.1.3-4.1 \times 10^{3}\right)$ and eosinophilia $\left(1.61 \times 10^{3}\right.$ eosinophils/ $\mu$ l; reference $\left.0-0.6 \times 10^{3}\right)$, with no anemia or thrombocytopenia. The biochemistry panel was normal with no hyperglobulinemia or hypoalbuminemia typical of canine L. infantum infection. ELISA serology was positive with an optical density (O.D.) of 1.88 (cut off 0.6 O.D.). Cytology of the lesion aspirate showed a large number of Leishmania amastigotes in macrophages and also outside cells presumably freed from breaking host cells during the aspiration (Figure 4). The culture taken by needle aspiration was positive for Leishmania promstigotes and ITS1-

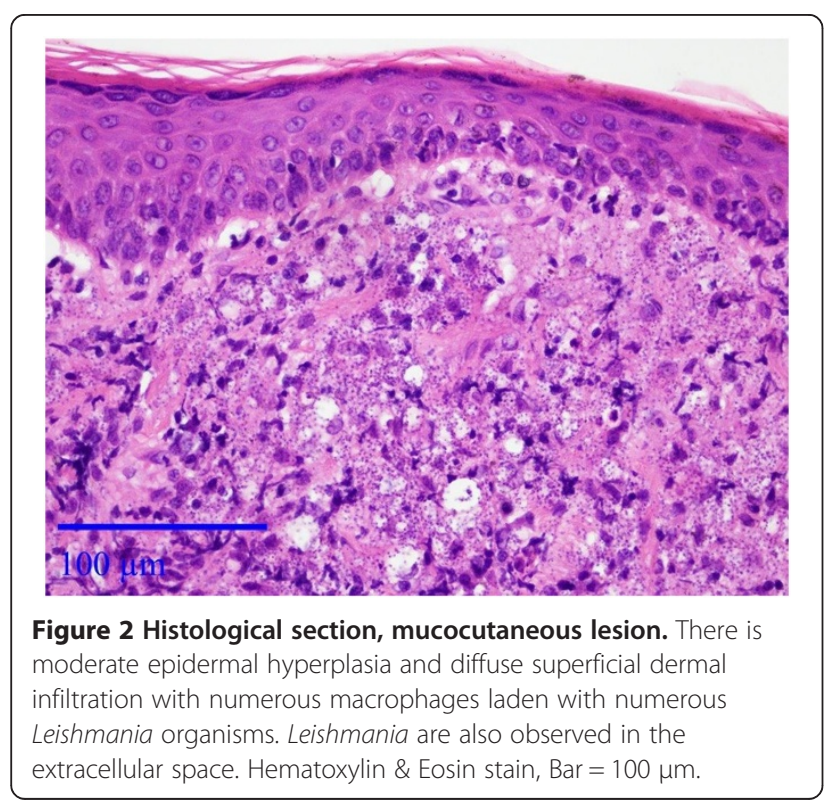

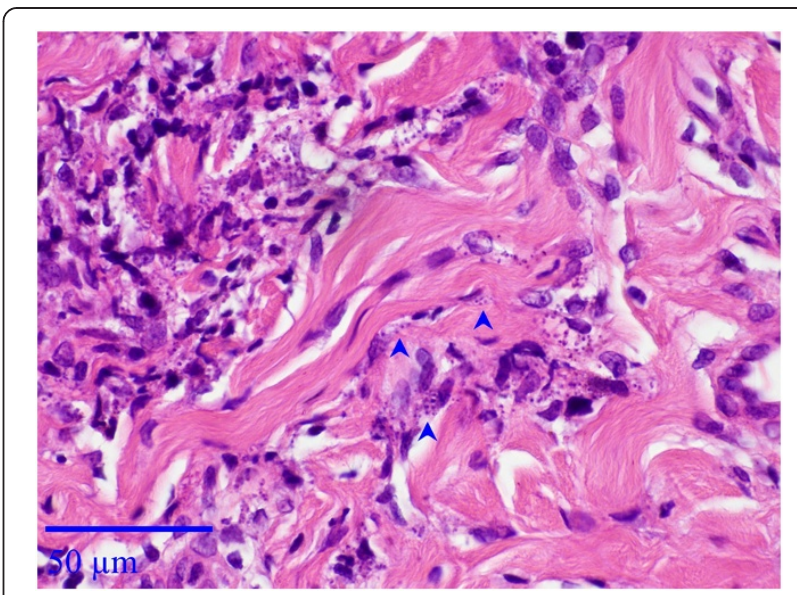

Figure 3 Histological section, mucocutaneous lesion, deep dermis. Leishmania amastigotes are both intrahistiocytic and extracellular. Note presence of Leishmania organisms in fibroblasts (arrowheads). Hematoxylin \& Eosin stain, Bar $=50 \mu \mathrm{m}$.

PCR-HRM was negative in blood and positive from the culture with an HRM curve compatible with L. tropica [3]. Sequencing of a 253 bp ITS1 PCR DNA product was carried out at the Hebrew University and the obtained sequence was deposited in GenBank [KF974365] and found by BLAST analysis (http://www.ncbi.nlm.nih. gov/BLAST) to be $100 \%$ identical to the closest match, L. tropica from Iran [KC505439], with 99\% coverage. An additional $400 \mathrm{bp}$ ITS1 fragment was amplified using primers ITS1F and ITS2R4 essentially as previously described [4] with some modification in the thermal profile, e.g. initial denaturation at $95^{\circ} \mathrm{C}$ for $5 \mathrm{~min}$, followed by 37 cycles each consisting of: $30 \mathrm{~s}$ at $95^{\circ} \mathrm{C}$ (denaturation),

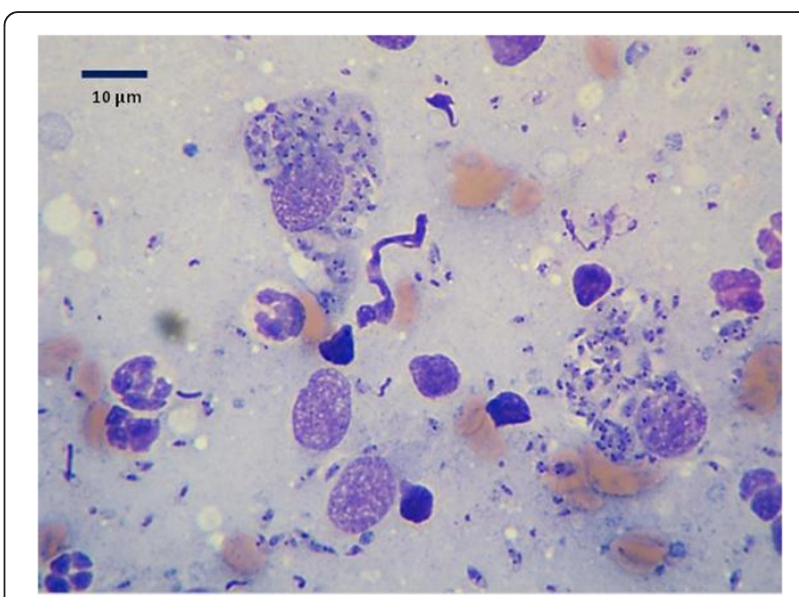

Figure $\mathbf{4}$ Cytology of lesion. Cytology of aspirate from the mucocutaneous lesion stained by May Grunwald Giemsa showing numerous intracellular Leishmania amastigotes and some

extracellular amastigotes persumably freed from the host cell during the aspiration. 


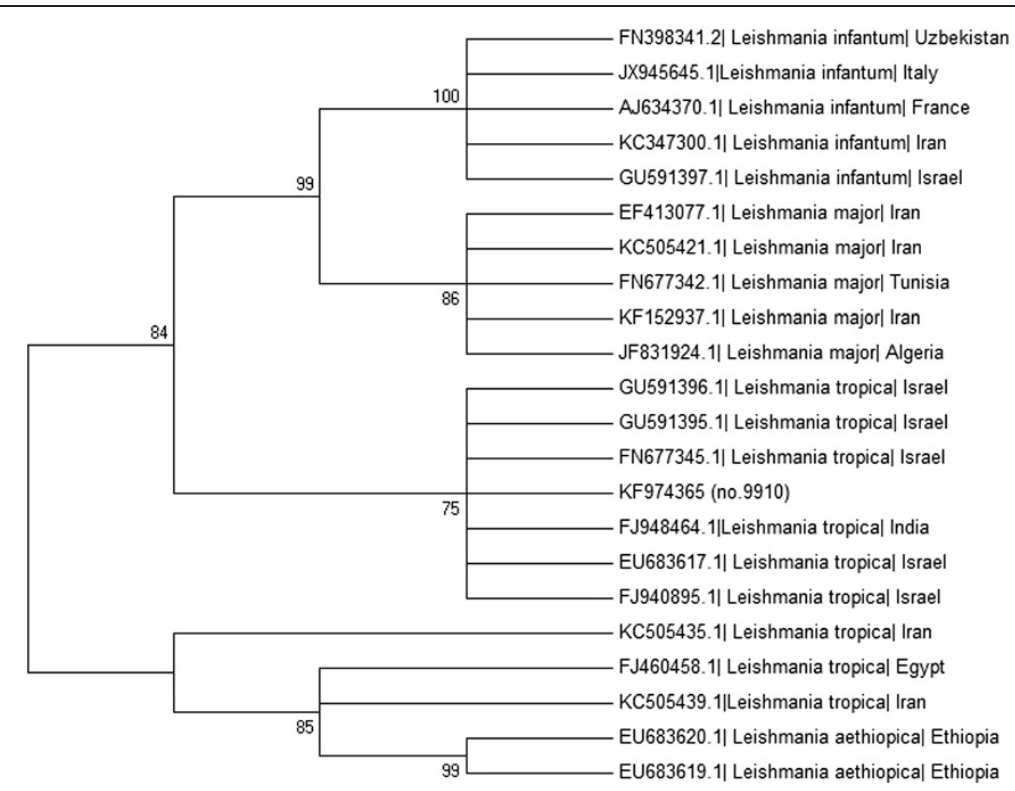

Figure 5 Phylogram. A Maximum Likelihood phylogram comparing 253 bp ITS1 rRNA DNA Leishmania sequences from the Israeli dog L. tropica strain [KF974365] with other L. tropica strains and Old World Leishmania spp. The GenBank accession numbers, Leishmania sp. and country of origin are included for each sequence.

$30 \mathrm{~s}$ at $58^{\circ} \mathrm{C}$ (annealing), and $90 \mathrm{~s}$ at $72^{\circ} \mathrm{C}$ (extension). After the last cycle, the extension step was continued for a further $5 \mathrm{~min}$. The $400 \mathrm{bp}$ ITS1 fragment was sequenced and submitted to GenBank [KJ010813]. The L. tropica isolate from the dog's lesion was maintained in culture and frozen down as strain LRC-L1647.

Phylograms of the 253 and 400 bp L. tropica ITS1 fragments [KF974365 and KJ010813] from the dog were constructed to compare these sequences to other $L$. tropica strains and additional Leishmania spp. present in GenBank. Sequences were analyzed using the MEGA version 5.1 (http://www.megasoftware.net) and phylograms were constructed using the Maximum likelihood algorithm with the Tamura-Nei model. Bootstrap replicates were performed to estimate the node reliability, and values were obtained from 500 randomly selected samples of the aligned sequence data (Figure 5 and Additional file 1:
Figure S1). The phylograms indicated that the dog's L. tropica sequences clustered together with other $L$. tropica strains from Israel and other Asian countries, separately from a second group of L. tropica strains which clustered together with $L$. aethiopica, and also away from $L$. major and $L$. infantum.

The dog was treated with allopurinol at $10 \mathrm{mg} / \mathrm{kg}$ every 12 hours as recommended for dogs infected with $L$. infantum [5]. One month after the initiation of treatment the dog's lesion was in a progressive state of healing, had considerably shrunk in size and appeared to be scarring. Two months after treatment initiation the lesion had almost disappeared and was replaced by depigmented skin and a small round scar (Figure 6). The owners reported that the dog was active and in good health. Repeat $\mathrm{CBC}$ and biochemistry panel were within normal limits except for a persisting lymphocytosis of 5.87 lymphocytes/ $\mu$ l and
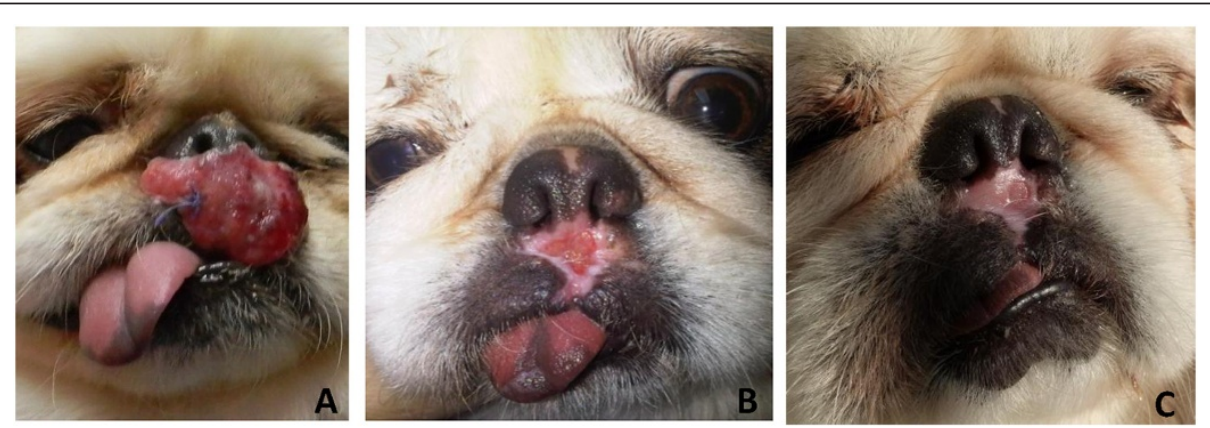

Figure 6 Treatment reposne. Response of lesion to allopurinol treatment (10 mg/kg every 12 hours). A- before treatment; B - 4 weeks after begining of treatement; $\mathbf{C}-8$ weeks after begining of treatment. 
eosinophilia of $1.28 \times 10^{3} / \mu \mathrm{l}$, which had decreased from the previous testing, in addition the ELISA serology O.D. decreased to 0.38 from 1.88 .

Ma'ale Adumim, the city where the dog described here lives, is a major emerging focus of human cutaneous leishmaniasis where 54 and $73 \mathrm{~L}$. tropica human cases were recorded during 2004 and 2005, respectively. These numbers reflect a sharp increase in the annual incidence from 2 cases per 100,000 residents from 1999 until 2003 to 214 per 100,000 residents in 2004 [6].

Leishmania tropica is an important causative agent of cutaneous leishmaniasis in the Old World including the Middle East, North Africa, Central Asia and some parts of southern Europe. It has also been described as a rare cause of human visceral leishmaniasis [7]. Although cutaneous leishmaniasis caused by $L$. tropica is usually considered an anthroponotic infection transmitted between people directly by phlebotomine sand flies without the involvement of an animal reservoir [8], in Israel, Jordan and the Palestinian Authority it is a zoonosis with the rock hyrax (Procavia capensis) as a main reservoir host $[9,10]$. Golden jackals (Canis aureus) and red foxes (Vulpes vulpes) have also been found to be infected with L. tropica in Israel and assumed to have a role in transmission of the infection between distant locations, but clinical signs of infection in these wild canids have not been detected [11].

In the domestic dog, L. tropica infection has been described in only a few cases reported from Morocco and Iran where infection was mostly described to involve visceral organs [12-16]. While some surveys described the detection of parasite infection from dog organs by culture or PCR without much detail on the manifestations of disease [14,16], reports from Morocco described two dogs infected with $L$. tropica with clinical manifestations similar to those found in canine L. infantum infection including generalized lymphadenomegaly, onychogryphosis, alopecia, keratoconjunctivitis, and also glomerulonephritis in one case [12,13]. A report from northwestern Iran also described $L$. tropica in a dog with cutaneous and visceral involvement comparable to canine $L$. infantum infection [17]. However, an earlier report from Morocco described seven dogs infected with $L$. tropica that had only dermal manifestations consisting of small facial papules without lymphadenomegaly or splenomegaly [18]. In addition, a five month old pup with multiple lesions adjacent to the eyes, lips and jaw described as mucocutaneous, as well as generalized lymphadenomegaly and visceral infection of the spleen and liver, was reported from central Iran [15].

The dog described in the current report was admitted with a distinctive presentation of a single large proliferative mucocutaneous lesion that contained a large amount of L. tropica amastigotes, which is different from the typical dermal manifestations of canine L. infantum infection and also from the descriptions of visceralizing L. tropica in dogs. The dog did not present with any manifestations of involvement of internal organs or hematological and serum biochemical abnormalities common in visceral infection such as anemia, hyperglobulinemia, or hypoalbuminemia, and there was no indication that infection had progressed beyond the skin as the blood PCR was negative, although such progression cannot be ruled out. The lymphocytosis and eosinophilia found by $\mathrm{CBC}$ are not common findings in canine leishmaniasis and could be explained by some unique inflammatory response with an eosinohilic and lymphocytic component. The seropositivity to $L$. infantum antigen was also reported in other cases of canine L. tropica infection $[12,13]$ and is not surprising as there is a strong serological cross-reactivity between different Leishmania species. None of the dogs described with $L$. tropica infection previously have been treated with anti-leishmanial drugs. The excellent initial response to allopurinol treatment and the healing of the dog's lesion indicate that allopurinol used as the major drug against canine $L$. infantum infection, is also effective against canine L. tropica infections.

\section{Conclusions}

This is the first report of focal mucocutaneous L. tropica infection in a dog and its response to anti-leishmanial treatment. Domestic and wild canine infection with $L$. tropica may be more prevalent in areas of endemic human L. tropica cutaneous leishmaniasis than currently recognized, and canines should be evaluated as possible additional reservoirs for human infection.

\section{Additional file}

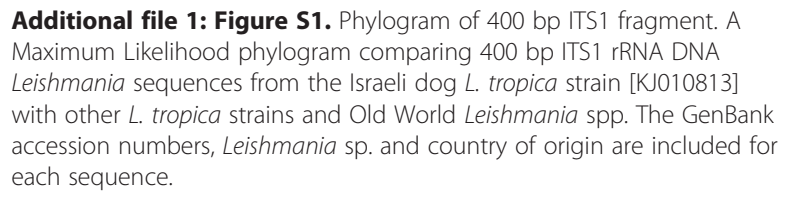

Competing interests

The authors declare that they have no competing interests.

\section{Authors' contributions}

GB collected the data, sampled the dog and wrote the manuscript; DZ is the veterinarian who saw the dog, biopsied it, administered treatment and continued to monitor its progress; YNB performed serology, PCR and DNA sequencing and performed the phylogenetic analyses; DYS isolated L. tropica and grew the parasite in culture; and AMB performed and interpreted the histopathology of the lesion. All authors read and approved the final version of the manuscript.

\section{Acknowledgments}

This study was supported by the Deutsche Forschungsgemeinschaft (DFG) as part of a German-Israeli-Palestinian cooperative project on the Emergence of Cutaneous Leishmaniasis in the Middle East: 'An investigation of Leishmania tropica in the Palestinian Authority and Israel' (SCHO 448/8-1). Publication of 
the CVBD9 thematic series has been sponsored by Bayer HealthCare - Animal Health division.

\section{Author details}

${ }^{1}$ School of Veterinary Medicine, Hebrew University, P.O. Box 12, Rehovot 76100, Israel. ${ }^{2}$ Maale Adumim Veterinary Clinic, Maale Adumim, Israel.

${ }^{3}$ Pathovet LTD, Yehosa Ben Hanania 81, Rehovot 76391, Israel.

Received: 31 December 2013 Accepted: 19 January 2014

Published: 24 March 2014

\section{References}

1. Hervás Rodríquez J, Mozos E, Méndez A, Pérez J, Gómez-Villamandos JC: Leishmania infection of canine skin fibroblasts in vivo. Vet Pathol 1996 33:469-473.

2. Baneth G, Dank G, Keren-Kornblatt E, Sekeles E, Adini I, Eisenberger CL, Schnur LF, King R, Jaffe CL: Emergence of visceral leishmaniasis in central Israel. Am J Trop Med Hyg 1998, 59:722-725.

3. Talmi-Frank D, Nasereddin A, Schnur LF, Schönian G, Töz SO, Jaffe CL, Baneth G: Detection and identification of old world Leishmania by high resolution melt analysis. PLOS Negl Trop Dis 2010, 4:e581.

4. Ajaoud M, Es-sette N, Hamdi S, El-Idrissi AL, Riyad M, Lemrani M: Detection and molecular typing of Leishmania tropica from Phlebotomus sergenti and lesions of cutaneous leishmaniasis in an emerging focus of Morocco. Parasit Vectors 2013, 6:217.

5. Solano-Gallego L, Miró G, Koutinas A, Cardoso L, Pennisi MG, Ferrer L, Bourdeau P, Oliva G, Baneth G: LeishVet guidelines for the practical management of canine leishmaniosis. Parasit Vectors 2011, 4:86.

6. Singer SR, Abramson N, Shoob H, Zaken O, Zentner G, Stein-Zamir C Ecoepidemiology of cutaneous leishmaniasis outbreak, Israel. Emerg Infect Dis 2008, 14:1424-1426.

7. Sacks DL, Kenney RT, Kreutzer RD, Jaffe CL, Gupta AK, Sharma MC, Sinha SP, Neva FA, Saran R: Indian kala-azar caused by Leishmania tropica. Lancet 1995, 345:959-961.

8. Ashford RW: The leishmaniases as emerging and reemerging zoonoses. Int J Parasitol 2000, 30:1269-1281

9. Jacobson RL, Eisenberger CL, Svobodova M, Baneth G, Sztern J, Carvalho J, Nasereddin A, El Fari M, Shalom U, Volf P, Votypka J, Dedet JP, Pratlong F, Schonian G, Schnur LF, Jaffe CL, Warburg A: Outbreak of cutaneous leishmaniasis in northern Israel. J Infect Dis 2003, 188:1065-1073.

10. Talmi-Frank D, Jaffe CL, Nasereddin A, Warburg A, King R, Svobodova M, Peleg O, Baneth G: Leishmania tropica in rock hyraxes (Procavia capensis) in a focus of human cutaneous leishmaniasis. Am J Trop Med Hyg 2010, 82:814-818

11. Talmi-Frank D, Kedem-Vaanunu N, King R, Bar-Gal GK, Edery N, Jaffe CL, Baneth G: Leishmania tropica infection in golden jackals and red foxes, Israel. Emerg Infect Dis 2010, 16:1973-1975.

12. Guessous-Idrissi N, Berrag B, Riyad M, Sahibi H, Bichichi M, Rhalem A: Leishmania tropica: etiologic agent of a case of canine visceral leishmaniasis in northern Morocco. Am J Trop Med Hyg 1997, 57:172-173.

13. Lemrani M, Nejjar R, Pratlong F: A new Leishmania tropica zymodemecausative agent of canine visceral leishmaniasis in northern Morocco. Ann Trop Med Parasitol 2002, 96:637-638.

14. Mohebali M, Hajjaran H, Hamzavi Y, Mobedi I, Arshi S, Zarei Z, Akhoundi B, Naeini KM, Avizeh R, Fakhar M: Epidemiological aspects of canine visceral leishmaniosis in the Islamic Republic of Iran. Vet Parasito/ 2005, 129:243-251.

15. Mohebali M, Malmasi A, Hajjaran H, Jamshidi S, Akhoundi B, Rezaei M, Janitabar S, Zarei H, Charehdar S: Disseminated Leishmaniasis Caused by Leishmania tropica in a Puppy from Karaj, Central Iran. Iran J Parasitol 2011, 6:69-73.

16. Hajjaran H, Mohebali M, Mamishi S, Vasigheh F, Oshaghi MA, Naddaf SR, Teimouri A, Edrissian GH, Zarei Z: Molecular identification and polymorphism determination of cutaneous and visceral leishmaniasis agents isolated from human and animal hosts in Iran. Biomed Res Int 2013, 2013:789326.
17. Hajjaran H, Mohebali M, Zarei Z, Edrissian GH: Leishmania tropica: another agent of canine visceral leishmaniasis in Iran. Iranian J Publ Health 2007, 36:85-88.

18. Dereure J, Rioux JA, Gallego M, Perières J, Pratlong F, Mahjour J, Saddiki H: Leishmania tropica in Morocco: infection in dogs. Trans $R$ Soc Trop Med Hyg 1991, 85:595.

doi:10.1186/1756-3305-7-118

Cite this article as: Baneth et al:: Mucocutaneous Leishmania tropica infection in a dog from a human cutaneous leishmaniasis focus.

Parasites \& Vectors 2014 7:118.

\section{Submit your next manuscript to BioMed Central and take full advantage of:}

- Convenient online submission

- Thorough peer review

- No space constraints or color figure charges

- Immediate publication on acceptance

- Inclusion in PubMed, CAS, Scopus and Google Scholar

- Research which is freely available for redistribution

Submit your manuscript at www.biomedcentral.com/submit
C Biomed Central 